\title{
Molecular Imaging of Prostate Cancer with PET
}

\author{
Hossein Jadvar
}

Molecular imaging is paving the way for precision and personalized medicine. In view of the significant biologic and clinical heterogeneity of prostate cancer, molecular imaging is expected to play an important role in the evaluation of this prevalent disease. The natural history of prostate cancer spans from an indolent localized process to biochemical relapse after radical treatment with curative intent to a lethal castrate-resistant metastatic disease. The ongoing unraveling of the complex tumor biology of prostate cancer uniquely positions molecular imaging with PET to contribute significantly to every clinical phase of prostate cancer evaluation. The purpose of this article was to provide a concise review of the current state of affairs and potential future developments in the diagnostic utility of PET in prostate cancer.

Key Words: correlative imaging; molecular imaging; oncology; PET; PET/CT; PET/MRI; radiopharmaceuticals; cancer; imaging; molecular; prostate

J Nucl Med 2013; 54:1685-1688

DOI: 10.2967/jnumed.113.126094

Prostate cancer remains a major public health problem in developed countries. The disease is characterized by evolution from a clinically localized hormone-naïve state to an eventually castrateresistant metastatic state (1). The widespread use of screening serum prostate-specific antigen (PSA) has resulted in downward stage migration and increased diagnosis of localized low-grade prostate cancers, leading to overdiagnosis and overtreatment (2). On the other hand, small high-grade tumors may be underdiagnosed and undertreated. There can be a high sampling error of about $40 \%$ with a standard transrectal ultrasound-guided biopsy procedure that may then lead to repeated biopsies (including saturation and transperineal biopsy) at still high sampling errors of up to $70 \%$ (3). Although localized disease is treated with curative intent, up to $35 \%$ of patients (or higher in select high-risk groups) may experience biochemical recurrence within a decade of primary therapy (4). Stratification of men with biochemical recurrence is pivotal in clinical decision making for prescribing and sequencing appropriate treatments that may include salvage therapy for local recurrence and systemic therapy for metastatic disease. When men develop a castrate-resistant state, the prognosis is poor and treatment is directed toward attempts to enhance survival and comfort.

Imaging can provide clinically important information at every phase of this heterogeneous disease. All major imaging modalities, including ultrasound, CT, MR imaging (including multiparametric

\footnotetext{
For correspondence or reprints contact: Hossein Jadvar, Department of Radiology, Keck School of Medicine of USC, University of Southern California, Los Angeles, CA 900033.

E-mail: jadvar@med.usc.edu

COPYRIGHT (C) 2013 by the Society of Nuclear Medicine and Molecular Imaging, Inc.
}

imaging and spectroscopy), and bone scintigraphy, have been used with documented strengths and limitations. PET is a quantitative imaging tool for interrogation of the underlying tumor biology. Several promising radiotracers are currently being investigated for PET imaging evaluation of prostate cancer including, but not limited to, ${ }^{18} \mathrm{~F}$-FDG; ${ }^{18} \mathrm{~F}$ - and ${ }^{11} \mathrm{C}$-choline; ${ }^{11} \mathrm{C}$-acetate; $16 \alpha-{ }^{18} \mathrm{~F}$-fluoro-5 $\alpha$ -dihydrotestosterone, targeted to the androgen receptor; anti-1amino-3- ${ }^{18}$ F-fluorocyclobutane-1-carboxylic acid, a synthetic L-leucine analog; and PET radiotracers based on prostate-specific membrane antigen (PSMA), prostate stem cell antigen, and gastrin-releasing peptide receptor (GRPR).

\section{GLUCOSE METABOLISM}

A hallmark of cancer is the Warburg effect of elevated glucose metabolism in malignant tissue relative to normal tissue. This forms the basis for increased accumulation of ${ }^{18} \mathrm{~F}$-FDG in cancer (5). However, ${ }^{18} \mathrm{~F}$-FDG uptake in tissue is not specific for cancer and benign conditions may also display high tracer localization. ${ }^{18} \mathrm{~F}-\mathrm{FDG}$ PET/CT is generally limited in the detection and initial staging of primary prostate cancer because many primary tumors are small, slow-growing, well-differentiated, and multiple with low-level tumor uptake that can overlap with that of normal tissue and coexisting benign prostatic hyperplasia. Poorly differentiated tumors and prostatitis both may demonstrate high uptake.

${ }^{18}$ F-FDG PET/CT may detect occult metastatic disease in a small number of men who present with biochemical recurrence. It has restricted utility for the detection of local recurrence in the treated prostate bed because of overlap of uptake between recurrent tumor and posttherapy tissue changes. Furthermore, high urine activity in the nearby bladder can obscure local tumor uptake (6). ${ }^{18}$ F-FDG PET/CT may be most useful in the evaluation of the extent and treatment response of castrate-resistant metastatic disease (7).

\section{LIPOGENESIS}

Malignancy-induced increased cellular membrane synthesis is the basis for imaging prostate cancer with ${ }^{11} \mathrm{C}$-acetate, ${ }^{11} \mathrm{C}$-choline, and ${ }^{18} \mathrm{~F}$-fluorocholine PET radiotracers $(8)$. Acetate is transported across the cellular membrane through monocarboxylate transporter and participates in the de novo synthesis of fatty acids from acetyl-CoA and malonyl-CoA through the action of fatty acid synthase, which is upregulated in prostate cancer (9). Choline enters the cell via choline transporters and is used for biosynthesis of phosphatidylcholine in the tumor cell membrane through malignancy-induced overexpression of choline kinase (9). Similar to ${ }^{18}$ F-FDG, uptake of radiolabeled acetate and choline derivatives by the primary prostate tumor may overlap with that of benign prostatic hyperplasia and normal prostate gland tissue.

${ }^{11} \mathrm{C}$-acetate may be useful for pelvic nodal staging and treatment planning in men with intermediate-risk (T2b-T2c or Gleason score 7 or PSA $10-20 \mathrm{ng} / \mathrm{mL}$ ) to high-risk (T3a or Gleason score 
$8-10$ or PSA $>20 \mathrm{ng} / \mathrm{mL}$ ) prostate cancer $(10) .{ }^{11} \mathrm{C}$-acetate may also be useful in the localization of tumor recurrence in men with biochemical failure, with a detection rate that tends to be positively associated with increasing serum PSA level (11).

Both ${ }^{11} \mathrm{C}$-choline and ${ }^{18} \mathrm{~F}$-fluorocholine have received much attention over the past several years, particularly in Europe and Japan, for imaging of men with prostate cancer. Recently, ${ }^{11} \mathrm{C}$ choline was approved by the Food and Drug Administration for production and use to detect recurrent prostate cancer at the Mayo Clinic in Rochester, Minnesota (12). ${ }^{11} \mathrm{C}$-choline has the advantage of low excreted urine activity if scanning is performed sufficiently early after radiotracer injection, whereas ${ }^{18} \mathrm{~F}$-fluorocholine has the advantage of longer half-life that facilitates regional tracer distribution without the need for on-site cyclotron and radiochemistry facilities.

A systematic review and metaanalysis by Umbehr et al. of ${ }^{11} \mathrm{C}$ choline and ${ }^{18} \mathrm{~F}$-fluorocholine PET for the initial staging of prostate cancer reported a pooled sensitivity and specificity, on a perpatient basis (10 studies, $n=637$ patients), of $84 \%$ (95\% confidence interval $[\mathrm{CI}], 68 \%-93 \%)$ and $79 \%(95 \% \mathrm{CI}, 53 \%-93 \%)$, respectively (13). In restaging patients with biochemical recurrence, the metaanalysis demonstrated, on a per-patient basis (12 studies, $n=1,055$ patients), a pooled sensitivity and specificity of $85 \%$ (95\% CI, 79\%-89\%) and 88\% (95\% CI, 73\%-95\%), respectively (13).

Evangelista et al. published a systematic review and metaanalysis (19 studies, including 12 studies for all sites of disease, 3 for lymph node metastases, and 4 for local recurrence, $n=1,555$ patients) of the use of choline PET and PET/CT in biochemical relapse of prostate cancer (14). This metaanalysis reported a pooled sensitivity of $85.6 \%(95 \% \mathrm{CI}, 82.9 \%-88.1 \%)$ and pooled specificity of $92.6 \%(95 \% \mathrm{CI}, 90.1 \%-94.6 \%)$ for all sites of disease (prostatic fossa, lymph nodes, and bone), a pooled sensitivity of $75.4 \%(95 \% \mathrm{CI}, 66.9 \%-82.6 \%)$ and pooled specificity of $82 \%$ (95\% CI, 68.6\%-91.4\%) for prostatic fossa recurrence, and a pooled sensitivity of $100 \%(95 \% \mathrm{CI}, 90.5 \%-100 \%)$ and pooled specificity of $81.8 \%$ (95\% CI, $48.2 \%-97.7 \%$ ) for lymph node metastases. In the latter case, the reported perfect pooled sensitivity for detection of lymph node metastases may have been overestimated given the small number of relevant publications included in the metaanalysis.

Overall, there is limited but promising evidence for the use of choline PET/CT to stage patients with untreated, high-risk prostate cancer. Relatively robust evidence suggests that choline PET/ CT can be helpful for the evaluation of locoregional and distant metastatic disease in men with biochemical relapse of prostate cancer with a detection rate that is positively associated with increasing PSA level, increasing PSA velocity, and decreasing PSA doubling time. The current recommendation is for considering choline PET/CT as the first-line diagnostic procedure in patients with biochemical relapse showing PSA levels greater than $1 \mathrm{ng} / \mathrm{mL}$, PSA velocity higher than $1 \mathrm{ng} / \mathrm{mL} / \mathrm{y}$, or PSA doubling time less than 6 mo (15).

\section{AMINO ACID TRANSPORT}

Anti-1-amino-3- ${ }^{18}$ F-fluorocyclobutane-1-carboxylic acid (anti${ }^{18} \mathrm{~F}-\mathrm{FACBC}$ ) is a synthetic nonmetabolized amino acid analog that accumulates in prostate cancer via overexpression of the ASC (alanine, serine, and cysteine) transport system and other amino acid transport systems (16). The early studies of anti- ${ }^{18} \mathrm{~F}-\mathrm{FACBC}$ in prostate cancer have been encouraging and demonstrate an advantage over ${ }^{111}$ In-capromab pendetide scintigraphy with relatively high tumor uptake and little urinary excretion of the tracer (Fig. 1) (17). A recent study suggested that anti- ${ }^{18}$ F-FACBC may be advantageous over ${ }^{11} \mathrm{C}$-choline for localization of disease in biochemical failure (18). On the basis of the early encouraging results, additional studies will be needed in a larger cohort of patients to decipher the exact role of anti- ${ }^{18} \mathrm{~F}-\mathrm{FACBC}$ in the imaging evaluation of patients with prostate cancer.

\section{ANDROGEN RECEPTOR}

${ }^{18} \mathrm{~F}$-fluoro- $5 \alpha$-dihydrotestosterone $\left({ }^{18} \mathrm{~F}\right.$-FDHT) is a labeled analog targeting the androgen receptor, which plays an important role in the development, growth, and maintenance of the prostate gland. Studies of comparisons to ${ }^{18} \mathrm{~F}-\mathrm{FDG}$ have suggested that there may be diverse metabolic phenotypes of castrate-resistant cancers (androgen receptor-predominant, glycolysis-predominant, or androgen receptor/glycolysis-concordant) and that ${ }^{18} \mathrm{~F}$-FDHT is probably suited as a pharmacodynamic response marker, rather than a treatment response marker, in the phase 1 clinical trials of new antiandrogenic drugs (19).

\section{PSMA}

PSMA is a type II cell surface transmembrane glycoprotein (also known as folate hydrolase I or glutamate carboxypeptidase II) that is upregulated in prostate cancer and provides a rational target for diagnostic imaging and directed therapy. PSMA does not have a known naturally occurring ligand, and despite what is implied from the label, it has a low degree of expression in the small intestine, proximal renal tubule, salivary glands, brain, and neovasculature of some nonprostate tumors (e.g., kidney, bladder, pancreas, lung). Investigators have developed and evaluated, in preclinical animal and pilot human studies, several radiolabeled ligands using various platforms that include antibodies or antibody fragments targeting the intracellular (binding to apoptotic or necrotic cells) or extracellular (binding to viable cells) motifs of the antigen. These PSMA-based radiotracers include ${ }^{89} \mathrm{Zr}$-desferrioxamine B (DFO)7E11; ${ }^{64} \mathrm{Cu}$-labeled aptamers; and ${ }^{11} \mathrm{C}-,{ }^{18} \mathrm{~F}-,{ }^{68} \mathrm{Ga}-,{ }^{64} \mathrm{Cu}-$, and

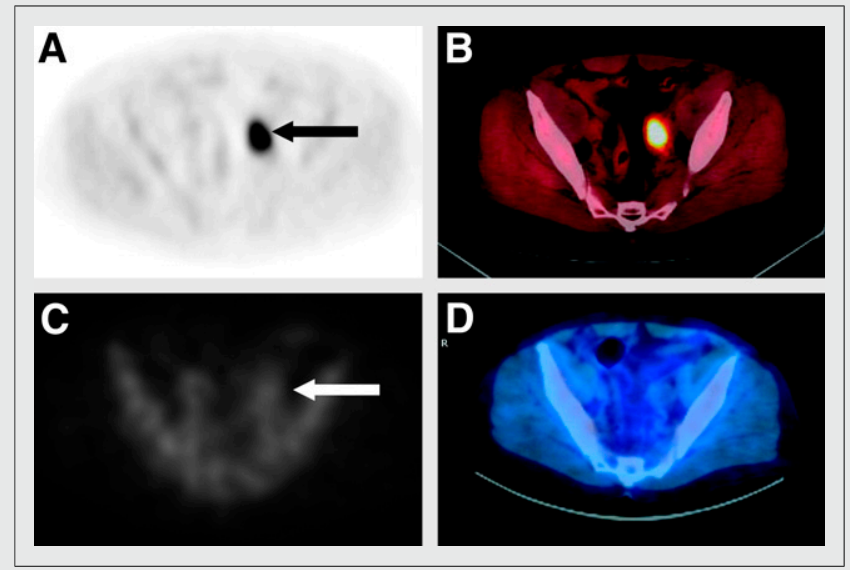

FIGURE 1. A 67-y-old man with biochemical recurrence of prostate cancer. Axial PET (A) and axial fused PET/CT (B) images show intense localization of anti-18 F-FACBC in metastatic left external iliac nodal basin (arrow). Axial SPECT (C) and axial fused PET/CT (D) demonstrate only low-grade activity on ${ }^{111}$ In-capromab pendetide scintigraphy. (Reprinted with permission of (26).). 


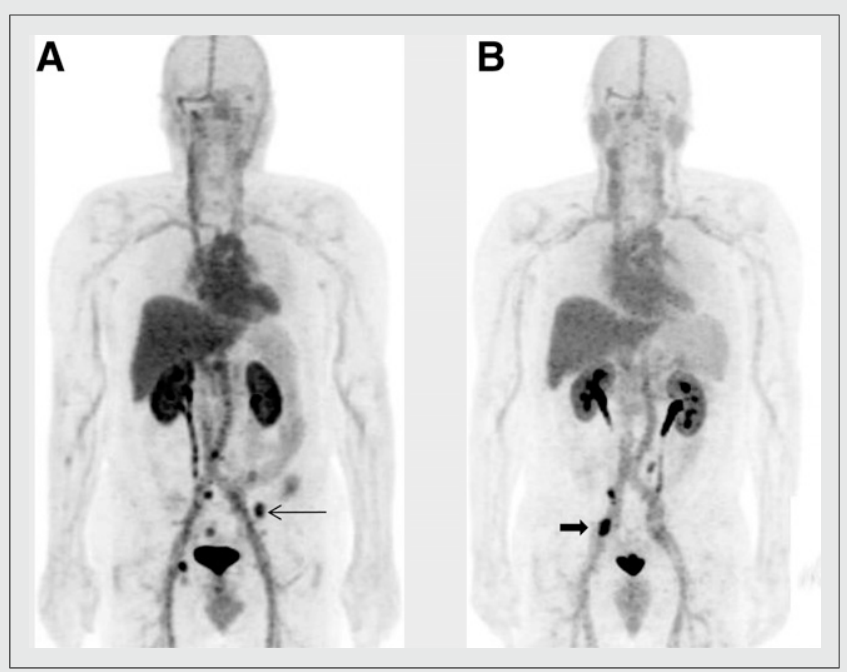

FIGURE 2. Anterior PET maximum-intensity-projection image obtained at $2 \mathrm{~h}$ after intravenous injection of ${ }^{18} \mathrm{~F}$-DCFBC $(\mathrm{N}$ $[N-[(S)-1,3-$ dicarboxypropyl]carbamoyl]-4-18 F-fluorobenzyl-L-cysteine) demonstrate normal biodistribution of this low-molecular-weight, urea-based inhibitor of PSMA and high focal accumulation (arrows) in bone (A) or nodal metastases (B) from prostate cancer. (Reprinted with permission of (27).).

${ }^{86}$ Y-labeled low-molecular-weight inhibitors of PSMA including ${ }^{18}$ F-labeled $N$-[N-[(S)-1,3-dicarboxypropyl] carbamoyl $]-4-\left[{ }^{18} \mathrm{~F}\right]$ fluorobenzyl-1-cysteine and ${ }^{18} \mathrm{~F}$-labeled phosphoramidate peptidomimetic with some formulations that can also be labeled with radionuclides such as ${ }^{177} \mathrm{Lu}$ or ${ }^{90} \mathrm{Y}$ for targeted radioimmunotherapy (Fig. 2) (20).

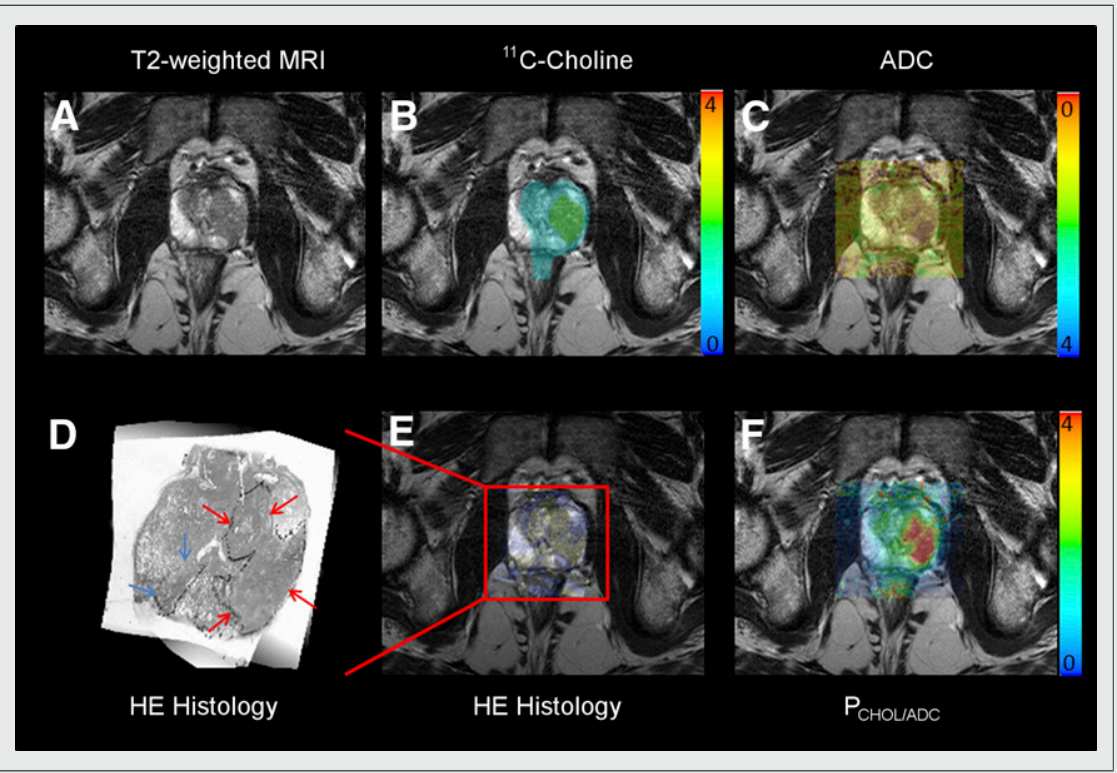

FIGURE 3. Fused multiparametric MR imaging and PET with ${ }^{11} \mathrm{C}$-choline in patient with true-positive high-grade (Gleason $4+3$ ) left lobe (red arrows) and false-negative lowvolume intermediate-grade (Gleason $3+3$ ) right lobe (blue arrows) prostate cancer. (A) T2-weighted MR image. (B) Fused ${ }^{11} \mathrm{C}$-choline PET and T2-weighed MR image. (C) Apparent diffusion coefficient map obtained from diffusion-weighted image coregistered over T2-weighted MR image. (D) Hematoxylin and eosin histology slice. (E) Histology slice coregistered over T2-weighted MR image. (F) Parametric fusion PET/MR image using $\mathrm{P}_{\mathrm{CHOLADC}}$ ratio defined as quotient of standardized uptake value over apparent diffusion coefficient values. $\mathrm{ADC}=$ apparent diffusion coefficient; $\mathrm{CHOL}=$ choline; $\mathrm{HE}=$ hematoxylin and eosin. (Reprinted with permission of (28).).

\section{BONE MATRIX}

The bone uptake of ${ }^{18} \mathrm{~F}-\mathrm{NaF}$ is related to chemisorption with exchange of ${ }^{18} \mathrm{~F}^{-}$ion for $\mathrm{OH}^{-}$ion on the surface of the hydroxyapatite matrix of bone to form fluoroapatite and migration of ${ }^{18} \mathrm{~F}^{-}$ into the crystalline matrix of bone. ${ }^{18} \mathrm{~F}-\mathrm{NaF}$ PET/CT has been to provide higher sensitivity and specificity than wection of osseous metastases in men with prostate cancer (21). The clinical use of ${ }^{18} \mathrm{~F}-\mathrm{NaF}$ PET/CT is projected to grow on the basis of increasing availability of PET/CT scanners, commercial regional distribution of ${ }^{18} \mathrm{~F}$-labeled radiotracers, lingering uncertainty on the Center for Medicare and Medicaid Services for scan reimbursem Center for Medicare and Medicaid Services for scan reimbursement through participation in the National Oncologic PET Registry.

\section{OTHER PROMISING BIOMARKERS}

Several other approaches are also in the preclinical stages of development and testing for molecular imaging of prostate cancer with PET. For example, ${ }^{68}$ Ga-DOTA-CHCO-Gly-4-aminobenzylGln-Trp-Ala-Val-Gly-His-Leu-Met-NH is a radiolabeled ligand based on bombesin, which is an amino acid analog of the human gastrin-releasing peptide that binds to the GRPR. GRPR is overxpressed in prostate cancer.

Researchers have also developed radiolabeled ligands targeting free PSA (e.g., ${ }^{89} \mathrm{Zr}-5 \mathrm{~A} 10$ monoclonal antibody), prostate stem cell igen (PSCA) (e.g., ${ }^{124}$ I-anti-PSCA antibody fragments), and thymidine kinase for assessing cellular proliferation and cellular (e.g., 1-(2'-deoxy-2'-fluoro-b-D-arabinofuranosyl)thymidine). There have also been reporter gene-reporter probe approaches for direct visualization and characterization of sentinel lymph nodes in prostate cancer using the reporter gene herpes simplex virus type 1 TK and reporter probe 9 -(4- ${ }^{18} \mathrm{~F}$-fluoro-3-[hydroxymethyl]butyl)guanine (22).

\section{POTENTIAL OPPORTUNITIES WITH PET/MR IMAGING}

PET/MR imaging provides combined structural, metabolic, and functional imaging information that can potentially affect patient management and outcome. It may also enhance patient convenience by providing a 1-stop diagnostic imaging work-up, reducing patient anxiety, total scan time, and recalls for repeated scanning (23). Simultaneous acquisition of multiparametric MR and PET images with an appropriate radiotracer may be particularly valuable for identifying highyield candidate biopsy sites that could reduce false-negative initial and repeated biopsies. Moreover, combined PET/MR imaging could facilitate the use of targeted focal therapy and the determination of its efficacy (Fig. 3) (24). Another interesting prospect is the potential utility of dual-modality imaging agents in this clinical setting (25).

\section{CONCLUSION}

PET with several radiotracers can assess the underlying altered biology of prostate 
cancer, including glucose metabolism, fatty acid metabolism, amino acid metabolism, DNA synthesis, and expression and activity levels of a variety of receptors, enzymes, and other cancerspecific and nonspecific biomarkers. The combination of PET with MR imaging, in view of the recent emergence of hybrid PET/MR imaging systems, may also provide additional opportunities for facilitating targeted biopsy, focal therapy, and 1-stop imaging localization of disease sites in patients with biochemical failure. Additional potential applications may include not only the assessment but also the deciphering of new insights into the biologic changes induced by various novel therapies. Molecular imaging with PET is therefore aligned seamlessly with the concept of precision and personalized medical care, which is applicable to all clinical phases of prostate cancer.

\section{ACKNOWLEDGMENT}

This work was supported by the National Institutes of Health, National Cancer Institute, grants R01-CA111613 and R21-142426. No other potential conflict of interest relevant to this article was reported.

\section{REFERENCES}

1. Schrecengost R, Knudsen KE. Molecular pathogenesis and progression of prostate cancer. Semin Oncol. 2013;40:244-258.

2. Moore AL, Dimitropoulou P, Lane A, et al. Population-based prostate-specific antigen in the UK leads to a stage migration of prostate cancer. BJU Int. 2009;104:1592-1598.

3. Presti J Jr. Does the yield of prostate cancer biopsy and repeat biopsy justify the frequency of their use? Nat Clin Pract Urol. 2008;5:246-247.

4. Bruce JY, Lang JM, McNeel DG, et al. Current controversies in the management of biochemical failure in prostate cancer. Clin Adv Hematol Oncol. 2012;10:716-722.

5. Hanahan D, Weinberg RA. Hallmarks of cancer: the next generation. Cell. 2011; 144:646-674.

6. Jadvar H, Desai B, Ji L, et al. Prospective evaluation of ${ }^{18} \mathrm{~F}-\mathrm{NaF}$ and ${ }^{18} \mathrm{~F}-\mathrm{FDG}$ PET/CT in detection of occult metastatic disease in biochemical recurrence of prostate cancer. Clin Nucl Med. 2012;37:637-643.

7. Jadvar H, Desai B, Ji L, et al. Baseline ${ }^{18}$ F-FDG PET/CT parameters as imaging biomarkers of overall survival in castrate-resistant metastatic prostate cancer. J Nucl Med. 2013;54:1195-1201.

8. Suburu J, Chen YQ. Lipids and prostate cancer. Prostaglandins Other Lipid Mediat. 2012;98:1-10.

9. Apolo AB, Pandit-Taskar N, Morris MJ. Novel tracers and their development for the imaging of metastatic prostate cancer. J Nucl Med. 2008;49:2031-2041.

10. Haseebuddin M, Dehdashti F, Siegel BA, et al. ${ }^{11} \mathrm{C}$-acetate PET/CT before radical prostatectomy: nodal staging and treatment failure prediction. $\mathrm{J} \mathrm{Nucl}$ Med. 2013;54:699-706.
11. Mohsen B, Giorgio T, Rasoul ZS, et al. Application of ${ }^{11} \mathrm{C}$-acetate positron emission tomography (PET) imaging in prostate cancer: systematic review and meta-analysis of the literature. BJU Int. June 13, 2013 [Epub ahead of print].

12. Mitchell CR, Lowe VJ, Rangel LJ, et al. Operational characteristics of ${ }^{11} \mathrm{C}$ choline positron emission tomography/computerized tomography for prostate cancer with biochemical recurrence after initial treatment. J Urol. 2013;189: 1308-1313.

13. Umbehr MH, Muntener M, Hany T, et al. The role of choline and ${ }^{18} \mathrm{~F}$-fluorocholine positron emission tomography (PET) and PET/CT in prostate cancer: a systematic review and meta-analysis. Eur Urol. 2013;64:106-117.

14. Evangelista L, Zattoni F, Guittilla A, et al. Choline PET and PET/CT and biochemical relapse of prostate cancer. Clin Nucl Med. 2013;38:305-314.

15. Castellucci P, Picchio M. ${ }^{11} \mathrm{C}$-choline PET/CT and PSA kinetics. Eur J Nucl Med Mol Imaging. 2013;40(suppl 1):S36-S40.

16. Okudaira $\mathrm{H}$, Nakanishi $\mathrm{T}$, Oka $\mathrm{S}$, et al. Kinetic analyses of trans-1-amino-3-[ $\left[{ }^{18} \mathrm{~F}\right]$ fluorocyclobutanecarboxylic acid transport in Xenopus laevis oocytes expressing human ASCT2 and SNAT2. Nucl Med Biol. 2013;40:670-675.

17. Schuster DM, Savir-Baruch B, Nieh PT, et al. Detection of recurrent prostate carcinoma with anti-1-amino-3- ${ }^{18} \mathrm{~F}$-fluorocyclobutane-1-carboxylic acid PET/ CT and ${ }^{111}$ In-capromab pendetide SPECT/CT. Radiology. 2011;259:852-861.

18. Nanni C, Schiavina R, Boschi S, et al. Comparison of ${ }^{18} \mathrm{~F}-\mathrm{FACBC}$ and ${ }^{11} \mathrm{C}-$ choline PET/CT in patients with radically treated prostate cancer and biochemical relapse: preliminary results. Eur J Nucl Med Mol Imaging. 2013;40(suppl 1): 11-17.

19. Fox J, Blanc E, Schoder H, et al. Diversity of biology in castrate resistant prostate cancer [abstract]. J Nucl Med. 2009;50(suppl 2):136P.

20. Mease RC, Foss CA, Pomper MG. PET imaging in prostate cancer: focus on prostate-specific membrane antigen. Curr Top Med Chem. 2013;13:951-962.

21. Beheshti M, Langsteger W, Fogelman I. Prostate cancer: role of SPECT and PET in imaging bone metastases. Semin Nucl Med. 2009;39:396-407.

22. Jadvar H. Molecular imaging of prostate cancer: PET radiotracers. AJR. 2012; 199:278-291.

23. Jadvar H, Colletti PM. Competitive advantage of PET/MRI. Eur J Radiol. June 18, 2013 [Epub ahead of print].

24. Wetter A, Lipponer C, Nensa F, et al. Simultaneous ${ }^{18} \mathrm{~F}$ choline positron emission tomography/magnetic resonance imaging of the prostate: initial results. Invest Radiol. 2013;48:256-262.

25. Glaus $\mathrm{C}$, Rossin $\mathrm{R}$, Welch $\mathrm{MJ}$, et al. In vivo evaluation of ${ }^{64} \mathrm{Cu}$-labeled magnetic resonance nanoparticles as a dual-modality PET/MR imaging agent. Bioconjug Chem. 2010;21:715-722.

26. Schuster DM Votaw JR, Nieh PT, et al. Initial experience with the radiotracer anti-1-amino-3- ${ }^{18}$ F-fluorocyclobutane-1-carboxylic acid with PET/CT in prostate carcinoma. J Nucl Med. 2007;48:56-63.

27. Cho SY, Gauge KL, Mease RC, et al. Biodistribution, tumor detection, and radiation dosimetry of ${ }^{18} \mathrm{~F}-\mathrm{DCFBC}$, a low-molecular-weight inhibitor of prostate-specific membrane antigen, in patients with metastatic prostate cancer. J Nucl Med. 2012;53:1883-1891.

28. Park H, Wood D, Hussain H, et al. Introducing parametric fusion PET/MR imaging of primary cancer. $J$ Nucl Med. 2012;53:546-551. 